\title{
Literature Research on Experimental Investigations of Automotive Turbocharger Rotordynamics
}

\author{
P. Sass ${ }^{1}$ \\ ${ }^{1}$ Széchenyi István University, Department of Internal Combustion Engines \\ Egyetem tér 1, 9026, Győr, Hungary \\ e-mail: sass.peter@ga.sze.hu
}

Abstract: Contemporary turbochargers are high-speed rotating devices, commonly supported by full floating bearings or semi-floating bearings. Depending on the size and operational speed of the turbocharger, rotordynamics significantly changes. Industrial turbochargers are operating below 20.000 RPM and their rotor weight is substantial, leading to quasilinear rotordynamics behaviour. By contrast, automotive turbochargers are rotating up to 300.000 RPM with non-linear rotordynamics characteristics. Due to this non-linearity, rotor movement is intense, and bearing load is dynamically changing all the time. The consequence is reduced lifespan of the turbocharger. In this paper, the effect of changing bearing clearances, as well as the differences between semi-floating and full floating bearing constructions will be described based on journal papers scientific publications on the topic. Also, the damping and whirling phenomenon inside a bearing system will be investigated and presented in a comprehensive literature research on automotive turbocharger rotordynamics.

Keywords: turbocharger, full-floating bearing, semi-floating bearing, rotordynamics, vibration diagnostics

\section{Introduction}

A turbocharger contains a turbine and a compressor wheel interconnected with a common shaft, which is in most cases supported by full floating bearings or semifloating bearings. The design of the bearing system and dimensions of the inner and 
outer clearances have significant effect on subsynchronous rotor vibrations. The turbocharger rotor system has eigenfrequencies below the synchronous rotation frequencies. Therefore, subsynchronous excitation can cause induced subsynchronous rotor vibrations, and it is important to know the characteristics of these in the vibration spectrum [1].

The aim of the author of this review paper is to collect and summarise the most substantial observations from scientific literature written on the topic of turbocharger rotordynamics, and to make conclusions about the remaining potential in the research of turbocharger vibrations, particularly focusing on those phenomena related to operation of the bearing system. The varying load on the bearings can cause reduced lifespan of the turbocharger, and also has an influence on its running quality. Therefore, research of the vibrations inside the turbocharger central housing plays an important role in application of automotive turbochargers.

\section{Rotordynamics of Small Turbochargers Supported on Floating Ring Bearings - Highlights in Bearing Analysis and Experimental Validation}

This article written by Luis San Andres, Juan Carlos Rivadeneira, Kostandin Gjika, Christopher Groves and Gerry LaRue was published by the American Society of Mechanical Engineers in the Journal of Tribology 2007 [4]. It sums up basic knowledge on the linear and non-linear rotrodynamics phenomena of automotive turbochargers. The authors are focusing on subsynchronous vibrations measured on a turbocharger cold gas test bench, and compare the measurements with numerical predictions. Luis San Andres and Juan Carlos Rivadeneira state, that subsynchronous vibrations are occurring due to the circumferential whirling of the oil inside the bearing clearance. Moreover, if the outer bearing clearance is increased compared to the inner oil film thickness, subsynchronous vibrations can be suppressed. However, in practice, this is only true at low operating speeds [2, 3, 4].

\subsection{Motivation}

As claimed by the authors, the size of the inner and outer bearing clearances are one of the most important turbocharger design parameters from rotordynamics point of view. The turbocharger can operate in three different critical states or modes. In the first critical rotational speed regime or mode, the conical rotor deflection pattern dominates with relatively large rotor shaft movement both at the compressor and turbine side. In the second mode of operation, the rotor behaviour shows cylindrical deflections, while in the third critical rotor speed regime or mode, the turbocharger shaft reaches flexible deformation states. In the first two vibration modes, the rotor has rather low eigenfrequencies. When the rotor system is properly balanced, the 
amplitudes of the subsynchronous vibrations can be as high as three times larger than the synchronous ones. It is possible, that frequencies of subsynchronous vibrations overlap the low eigenfrequencies of the rotor system during rigid rotor modes because of the low eigenfrequencies of the system in this frequency range. The presented study and related references contain experimental and computational investigations on the effect of bearing clearance change on rotor vibrations $[4,5]$.

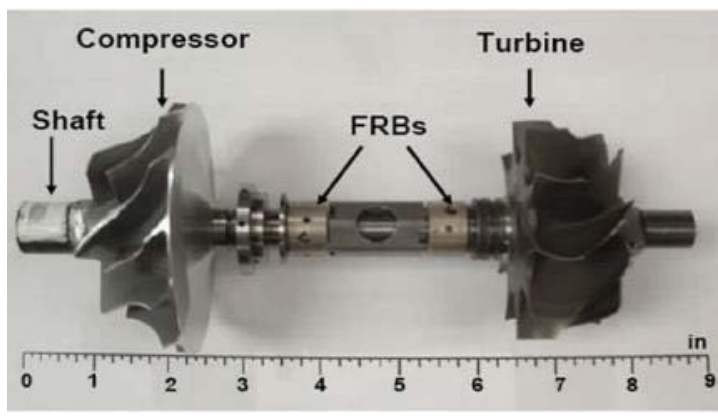

Figure 1. Measured turbocharger rotor of [4]

\subsection{Experimental turbocharger and shaft motion measurements}

The vibrations of the rotor were measured both on the compressor and on the turbine side. The most interesting part of the investigation was when rotor speed reached 65,000 $R P M$, and the floating bearing probably stopped spinning, which led to significant increase in the vibration amplitudes at about 0.5 times of the synchronous frequency $[4,6]$.

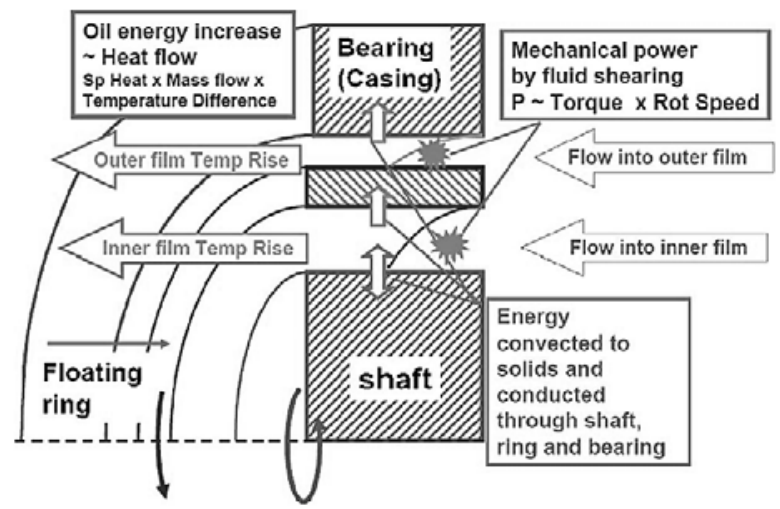

Figure 2. Heat flow inside the bearing housing [4] 
In real operating conditions and also on hot gas test benches, the shaft is a heat source for the bearing system, so the inner oil film heats up and the amplitudes of vibration could change due to the smaller damping coefficient because of this temperature increase. The measurements took part with oil pressures between 1,5 and 2,7 bar and oil temperatures between $32-46^{\circ} \mathrm{C}$, with rotor speeds up to 80,000 RPM. The shaft movement was also measured with inductive proximity sensors installed perpendicularly to each other. The turbocharger speed was measured on the compressor side with an infrared wheel-speed sensor. In addition, two optical fibre cables were built into the turbocharger center-housing facing exactly to the bearings to follow rotational of the bearings by detecting the oil threads passing by. With this measuring system, a relation could be found between the amplitude growths of the subsynchronous vibrations and rotational speed of the full floating bearing [4].

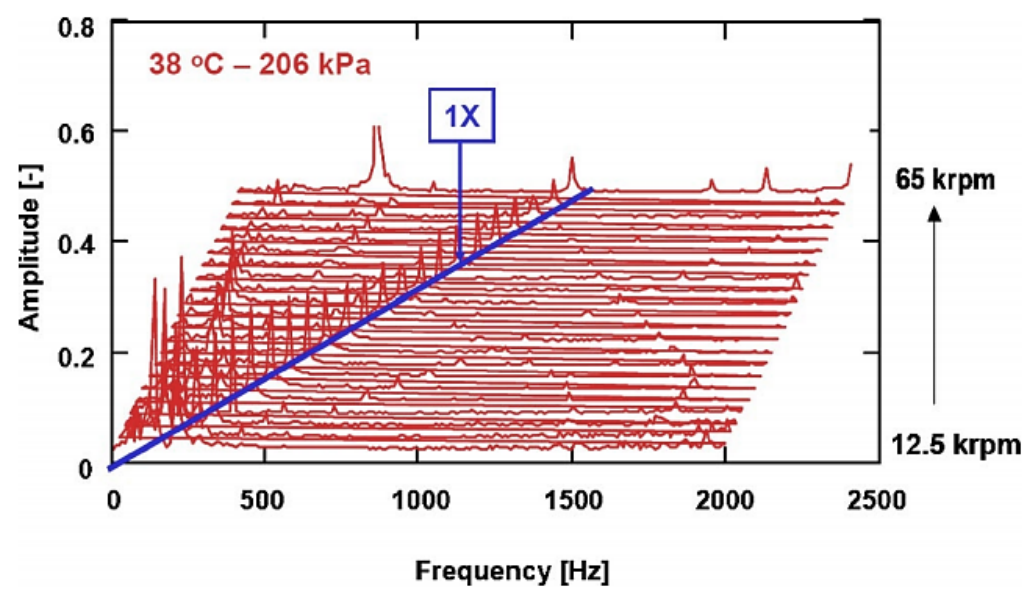

Figure 3. Waterfall diagram of the turbocharger of [4]

Below 20,000 RPM the band structure of the spectrum is very complex, and the vibration amplitudes are also high due to self-induced vibrations. The frequencies are close to the natural frequencies of the rotor at low operational speed.

At 65,000 RPM, a significant amplitude peak has become visible. It is a plausible assumption that this critical frequency could exactly be the rotational speed where the floating ring bearing stops spinning [4, 7]. At high rotational speed, the thickness of the inner oil film increases, while the outer film thickness decreases due to heat exchange effects. This assumed to be the reason why the movement of the floating bearing could get obstructed $[4,8]$. However, tests show that rotational speed of the floating bearing does not match well with the theoretically estimated values 


\section{Effects of bearing outer clearance on the dynamic behaviour of the full floating ring bearing supported turbocharger rotor}

The paper was published by Elsevier, in the journal of Mechanical Systems and Signal processing in 2012 [10], written by L. Tian, W.J. Wang and Z.J. Peng in the University of Sussex, United Kingdom.

\subsection{Motivation}

The document is dealing with the nonlinear rotordynamic phenomena by using run-up and run-down simulation methods. The authors investigate the effect of bearing outer clearance change on rotordynamic characteristics. With the numerical method applied, the natural frequencies of the rotor can be predicted and also the nonlinear jumps in the vibrations can be explained $[9,10]$.

\subsection{The Turbocharger Campbell diagram}

The Campbell diagram or also called as interference diagram shows the natural frequencies of a rotor system as a function of rotational speed.

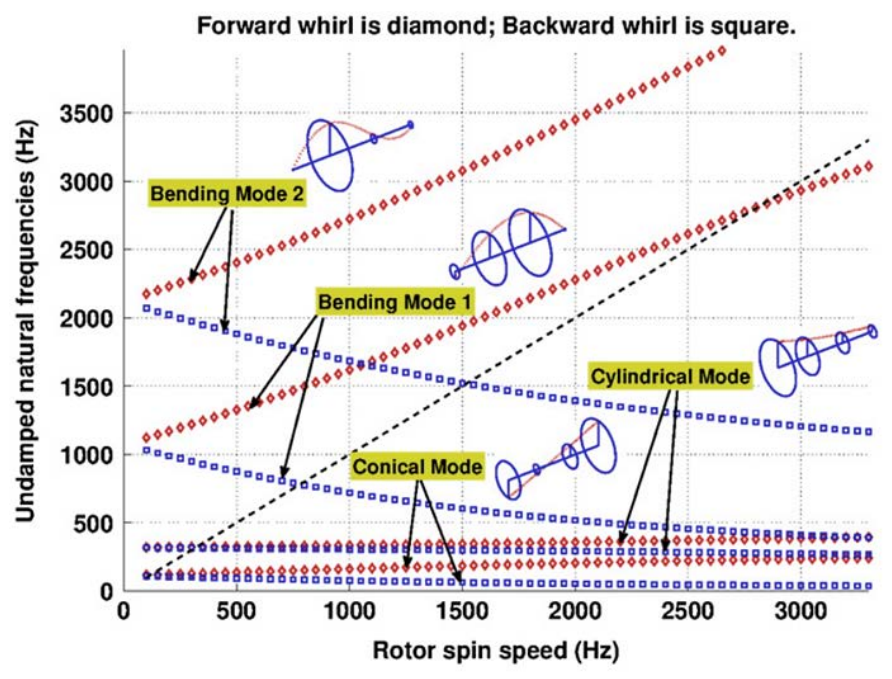

Figure 4. A turbocharger Campbell diagram (dash line is the synchronous line) [10]

Figure 4 shows the Campbell diagram of a turbocharger. When the rotational speed of the rotor is low, the subsynchronous (or even the synchronous) vibrations 
can match the natural frequencies of the system, which leads to self-induced vibrations. These self-induced vibrations could be critical and important to verify during the design phase of the turbocharger because of their large amplitudes and high acoustic emission levels [10, 11, 12].
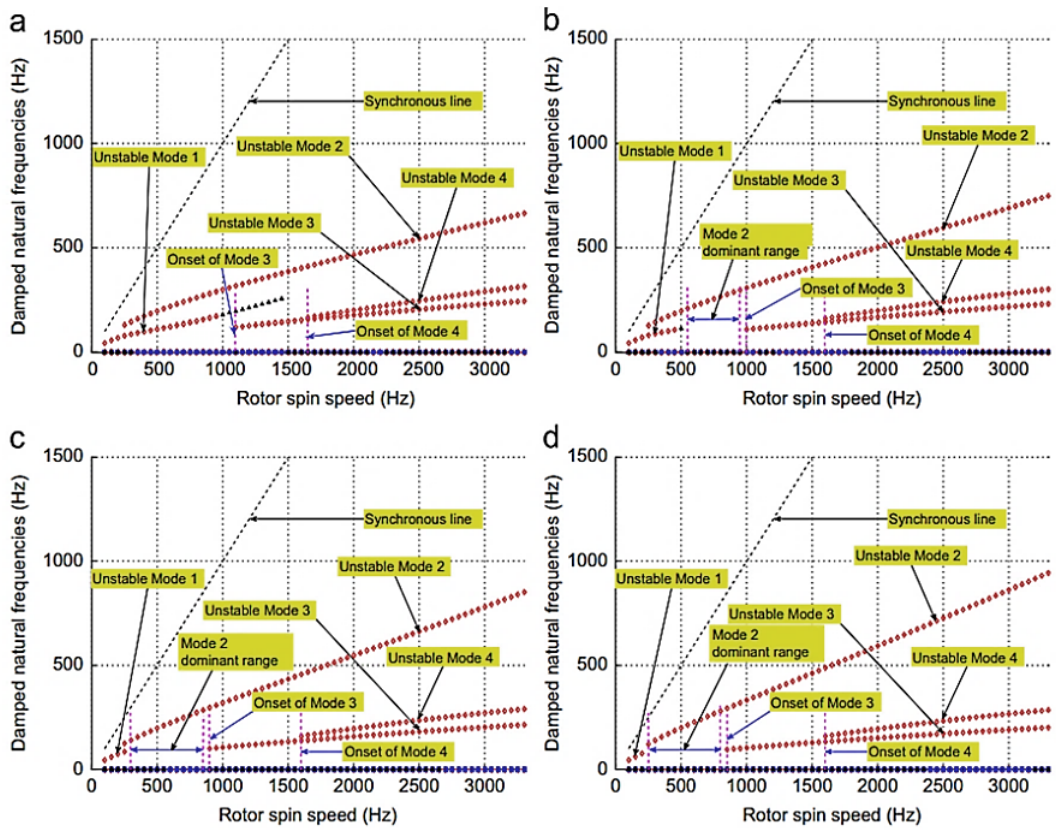

Figure 5. Campbell diagrams with varying bearing outer clearances $(a=30 \mu \mathrm{m} ; b=35 \mu \mathrm{m} ; c=40 \mu \mathrm{m} ; d=44 \mu \mathrm{m})$ [10]

Figure 5 shows, the effect of changing the bearing outer clearance. By increasing the outer clearance, the duration of the first unstable mode (Mode 1) can be significantly reduced, depending also on rotational speed. With $30 \mu \mathrm{m}$ outer clearance, the first unstable mode is visible till 60,000 RPM rotor speed. However, when the clearance is increased up to $44 \mu \mathrm{m}$, this first unstable mode lasts till $12,000 R P M$ only $[13,14]$.

\subsection{Result of the run-up simulations}

The author performed run-up simulations and evaluated the data in waterfall diagrams. The aim of the simulations was the investigation of non-linear subsynchronous vibrations [10]. 

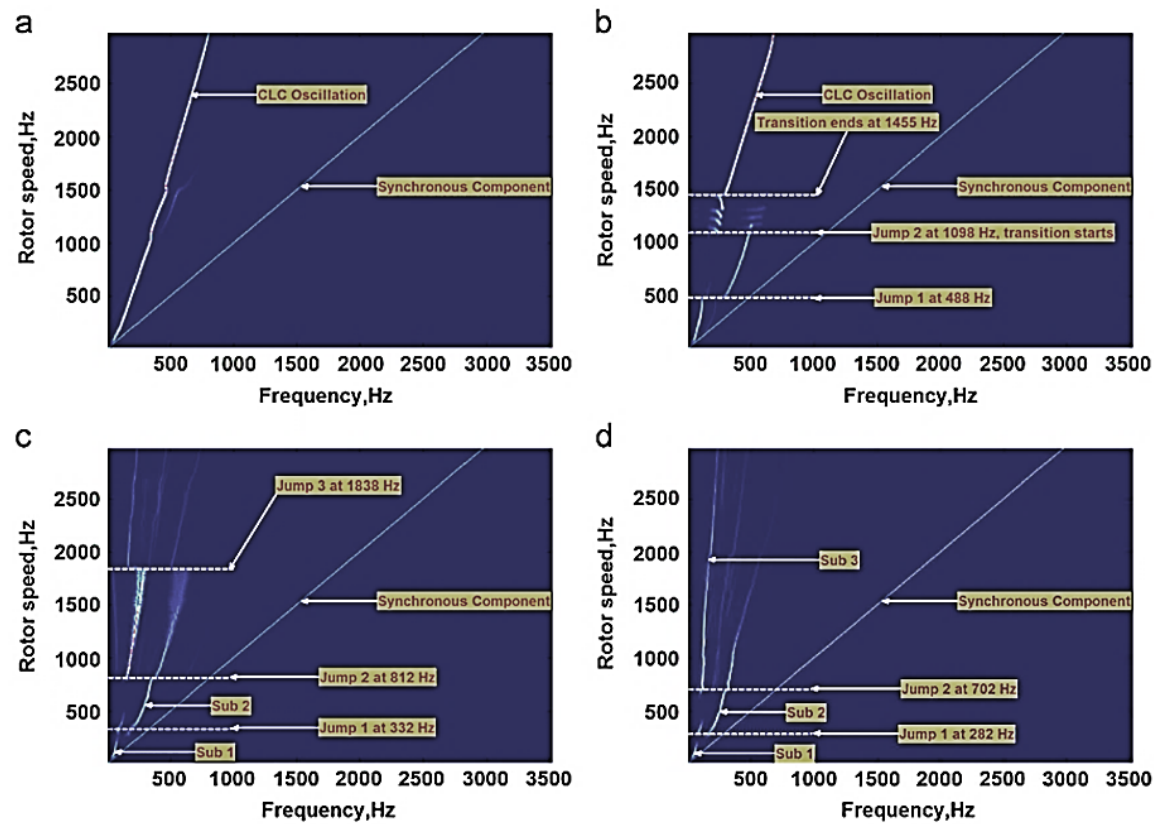

Figure 6. Waterfall diagrams from run-up simulations with $5 \mu$ m unbalance offset and varying outer bearing clearences from $30 \mu \mathrm{m}$ to $44 \mu \mathrm{m}$ (in the order of a to d) [10]

The simulation results show that the considered bearing outer clearance content can be subdivided into four ranges. The first range is from 30-33 $\mu \mathrm{m}$. In this clearance range nonlinear jump cannot be observed. In range 2, from 34-39 $\mu \mathrm{m}$ outer cleareance, nonlinear jumps are identified in a sequence of Sub 1 to Sub 2. In case of run-up simulations with bigger outer clearance, nonlinear jumps are likely to happen at lower rotational speeds. In range 3, where the clearance is $40-43 \mu \mathrm{m}$, the jump sequence is Sub 1 to Sub 2, then Sub 2 to Sub 1, and then Sub 1 to Sub 3. The jump speed also shifted to lower rotational speeds than in the previous range. Range 4 is with clearance from 44 to $50 \mu \mathrm{m}$. Non-linear jumps are also visible, following the sequence of Sub 1 to Sub 2, and then Sub 2 to Sub 3. Bigger bearing clearance leads to an earlier upset of Sub 2 vibration $[9,10,15]$. 


\section{Experimental and numerical investigations on an automotive turbocharger with a transparent bearing section}

This following paper is written by W. Köhl from Technical University of Darmstadt, M. Kreschel and D. Filsinger from IHI Charging Systems International GmbH, Germany in 2014 [16].

\subsection{Motivation}

In this publication the authors present a research on the dynamic behaviour of a full-floating radial bearing supported turbocharger via numerical investigations and measurements on a cold gas turbocharger test bench. The aim of this work is to examine changes in the orbit and deflections of the turbocharger rotor as bearing housing is being modified. The bearing housing itself was made of a special transparent material, and a high-resolution camera recorded the rotation movement of the bearing during test operation $[16,17]$.

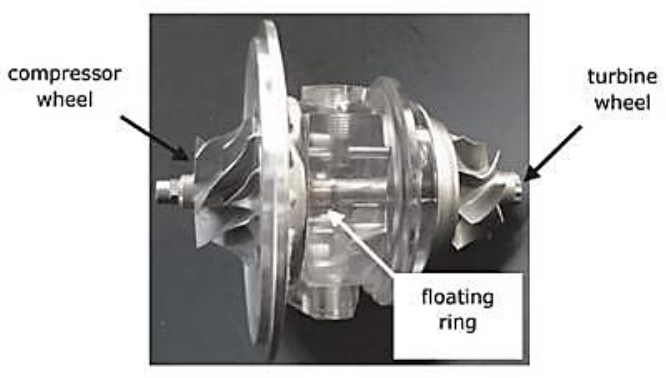

Figure 7. Turbocharger with a transparent bearing section [16]

\subsection{Highlights of the experimental setup}

The oil induced vibrations are most likely to be manufested as a "constant tone" noise from the charger. During the operation of the rotor, the frequency of subsynchronous vibrations is only changing to a small extent. The bearing system transmit these vibrations, and in the worst case and depending on the resonance frequencies, the turbine wheel, the compressor wheel or even the exhaust system could behave as a sound amplifier [18].

The measurements presented took part on a turbocharger cold gas test-bench, with $27^{\circ} \mathrm{C}$ oil temperature. The turbocharger speed was measured at the compressor wheel side, and the received signal was recorded through a low-pass filter. The configuration of the test can be seen in details in Table 1 below [16]. 


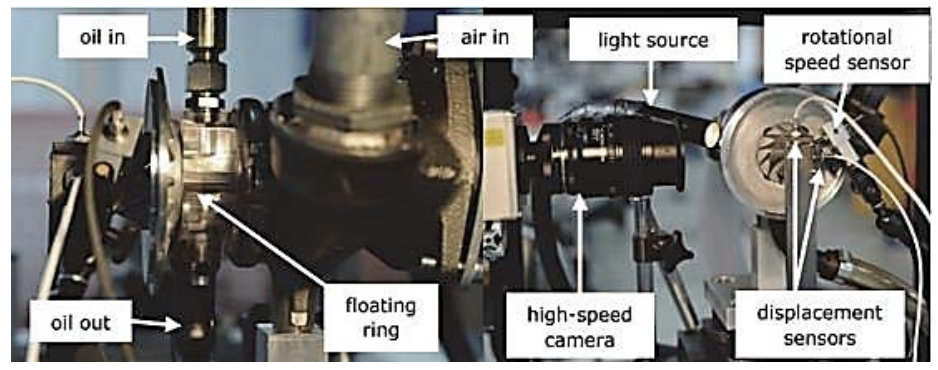

Figure 8. Setup of the measuring system of [16]

The essence of the investigation was to validate the numerical calculations with test-bench measurements, that is, to confirm that the calculated natural frequencies of the system are consistent with the measured ones. During the tests, the turbocharger performed a run-down operation from a specified rotational speed [16], instead of a run-up phase of some other investigations.

Table 1. Measurement setup details [16]

\begin{tabular}{|c|c|c|}
\hline Component & Type & Configuration \\
\hline Turbocharger & $\begin{array}{c}\text { IHI Charging Systems } \\
\text { International GmbH }\end{array}$ & $\begin{array}{c}\text { Waste gate closed } \\
\text { No compressor } \\
\text { housing }\end{array}$ \\
\hline High-Speed camera & $\begin{array}{c}\text { Photron: Fastcam } \\
\text { Ultima } 512\end{array}$ & $\begin{array}{c}32,000 \text { fps } \\
512 \times 12 \text { pixels }\end{array}$ \\
\hline Light source & $\begin{array}{c}\text { Shot: cold light } 2500 \\
\text { LCD }\end{array}$ & \\
\hline Displacement sensor & $\begin{array}{c}\mu \text {-Epsilon, Eddy } \\
\text { current DT 110-T-S1- } \\
\text { M-C3 }\end{array}$ & \\
\hline DSpace & & $\begin{array}{c}\text { Sampling } \\
\text { frequency: } 8192 \\
\mathrm{~Hz} \\
\end{array}$ \\
\hline Oil & ESSOLUBE X2 20W & $\begin{array}{c}\text { ๆoil }\left(27^{\circ}\right) \approx 110 \\
\text { mPas }\end{array}$ \\
\hline
\end{tabular}

\subsection{Numerical and experimental results}

As described by the authors, the system reaches its first natural frequency when the amplitudes of the subsynchronous vibrations originating from the inner bearing clearance reach their maximum and then begin to decrease. In addition, the rotational 
speed of the bearing also has an effect on the vibrations from the inner bearing clearance [16].

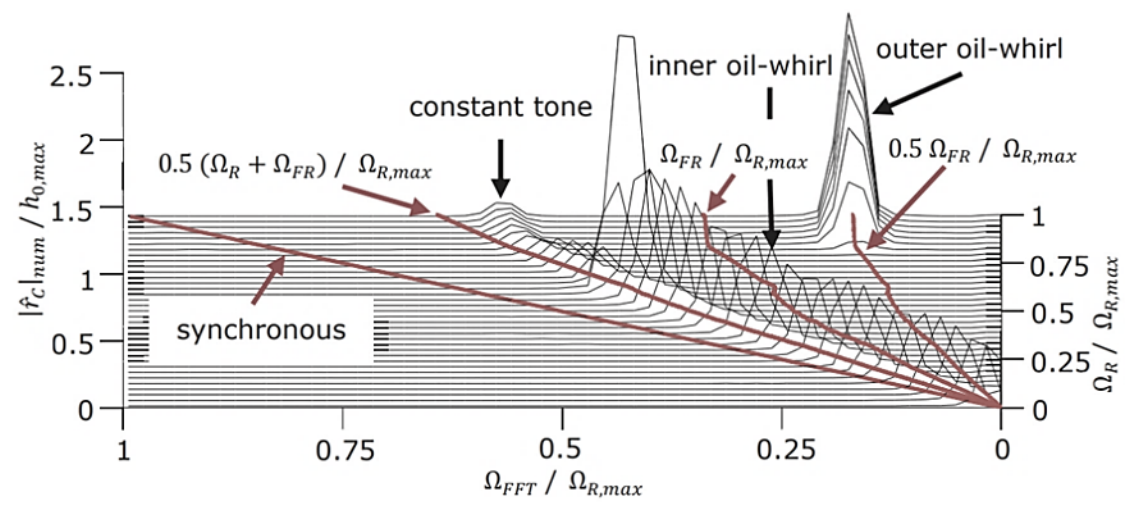

Figure 9. Calculated waterfall diagram from the numerical model [16]

On the calculated waterfall diagram of Figure 9., a significant amplitude peak appears from $0.8 \Omega(R, \max )$ with approximately half the frequency of the floating ring's rotational speed. This vibration belongs to the oil-whirl of the outer film, and does confirmed by the experiment. In general, the calculated rotational speed of the floating ring is higher than the experimentally detected floating ring speed $[16,19]$.

The authors wanted to adjust the computed results closer to the experimentally measured values, so the stiffness and the damping of the inner and outer oil film was modified in the model accordingly.

As predicted by the Campbell diagrams fom calculations, the effect of subsynchronous vibrations can be reduced if the floating ring rotational speed is reduced. The floating ring rotational speed can be decreased by changing the bearing geometry. If the inner bearing clearance increases, the stiffness of the inner oil film reduces. Thus, the constant tone phenomenon will likely occur at lower rotor speeds, causing smaller vibration amplitudes. To reduce the outer whirl, the damping of the outer oil film has to be increased [16, 20].

The rotational speed of the floating bearing was recorded, and the results show that the ring speed ratio $(\Omega$ fring $/ \Omega$ Rotor $)$ is in the relative speed range of $0.1 \ldots$ 0.2 . The friction torque, which drives the floating ring turned out to be smaller in reality than the numerically predicted values. This is caused by the different temperatures of the inner and outer oil film, which changes the viscosity of the oil. The transparent bearing housing and a high-speed camera allows to measure the rotational speed of the floating ring. With this help, the numerical models can be 
validated. The numerically calculated ring speed ratios are overestimated compared to the experiment. This could be improved by re-running the tests with oils of different viscosity levels [16, 19, 20].

\section{Effects of Semi-floating Ring Bearing Outer Clearance on the Subsynchronous Oscillation of Turbocharger Rotor}

The research presented in this section was published in the Chinese Journal of Mechanical Engineering written by Liang Feng, Zhou Ming, and Xu Quanyong, in the School of Aerospace Engineering, Tsinghua University, Beijing in 2016 [21].

\subsection{Motivation}

The paper presents research results on the topic of vibration diagnostics of turbochargers supported with semi-floating bearings. The outer clearance of the semi-floating bearing affects the magnitude and the frequency of the whirl motion phenomenon, which is important from turbocharger designs with semi-floating bearings perspective. To explore the effects of the outer clearance, the authors used a transient finite element analysis program for the rotor. The oil film bearing of the test was built and validated by another published experimental study.

The authors present that as the outer clearance increases, three subsequent steps are going to occur to the low-frequency subsynchronous vibrations. First, the vibration amplitudes are rather high, then a gradual amplitude recession happens. and finally, the two subsynchronous vibrations combine. The high-frequency subsynchronous oscillations start to evolve gradually first, then their amplitudes increase, and finally the vibration modes combine. The increasing outer clearance causes a „zipper” effect to these oscillations, which means that the subsynchrons vibrations start to combine at lower speeds if the outer clearance increases [21, 22, 23].

\subsection{Numerical Method}

The equivalent simplified rotor model was divided into 25 one-dimensional elements with 26 nodes. The blue section on the figure represents the aluminium alloy compressor impeller [21]. 


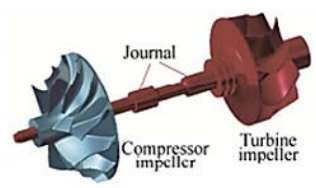

(a) Original rotor

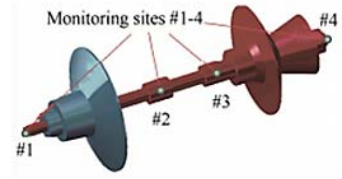

(b) Simplified rotor

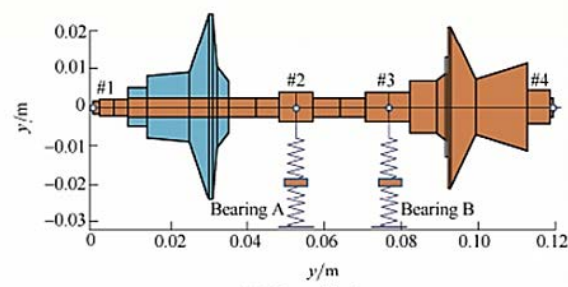

(c) FE model of rotor

Figure 10. Simplified model of the rotor of [21]

The basic rotordynamic equation, which helps to specify the correct model, is the following:

$$
\mathrm{M}_{\text {rotor }} \ddot{\mathrm{q}}_{\text {rotor }}+\left(\mathrm{C}_{\text {rotor }}+\Omega \mathrm{G}_{\text {rotor }}\right) \dot{\mathrm{q}}_{\text {rotor }}+\mathrm{K}_{\text {rotor }} \mathrm{q}_{\text {rotor }}=\mathrm{F}_{\text {rotor }} \text {, }
$$

In many turbochargers, the rotor is supported by one semi-floating ring, which incorporates two load surfaces (compressor side and turbine side). The ring can be considered as a rigid body since it is stubbier than the shaft itself [24].

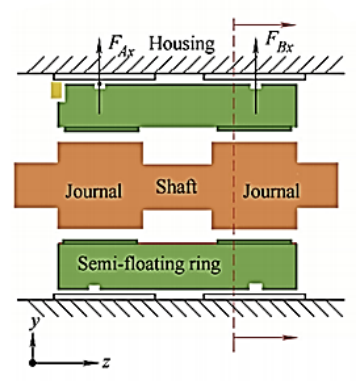

(a) Forces on the ring

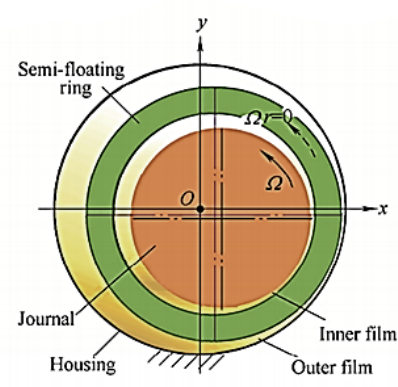

(b) Oil films position

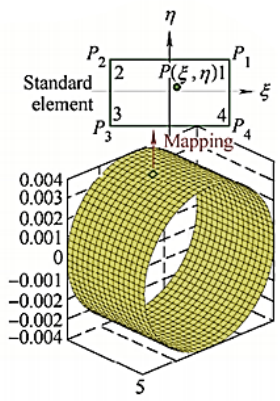

(c) Grids of oil film

Figure 11. Bearing model of [21]

For a comprehensive model, the oil film model also had to be deduced. The pressure distribution in a fluid film bearing can be described by the Reynolds equation. By solving the equations, the pressure at each node can be calculated and integrated into the nonlinear oil film force components that are then applied to the rotor-ring equation in each step of the numerical integration [21, 25]. 


\subsection{Non-linear analysis of ideal conditions}

Under ideal conditions, the gravity is neglected in the simulation, and the rotor is supposed to be perfectly balanced. The equilibrium position of the ring is the center of the housing, which position is unstable. The vibrations originating from the whirling motion of the oil will be visible in less than half of the rotor rotational speed. Figure 12 shows the decomposed limit cycles of the shaft orbit at different rotational speeds for the subsynchronous vibrations. The vibration amplitude at the compressor side is larger than the turbine side for Sub A, but for sub B the amplitudes for both sides are close. This may be caused by the different mass densities of the compressor wheel and the turbine wheel $[21,26]$.

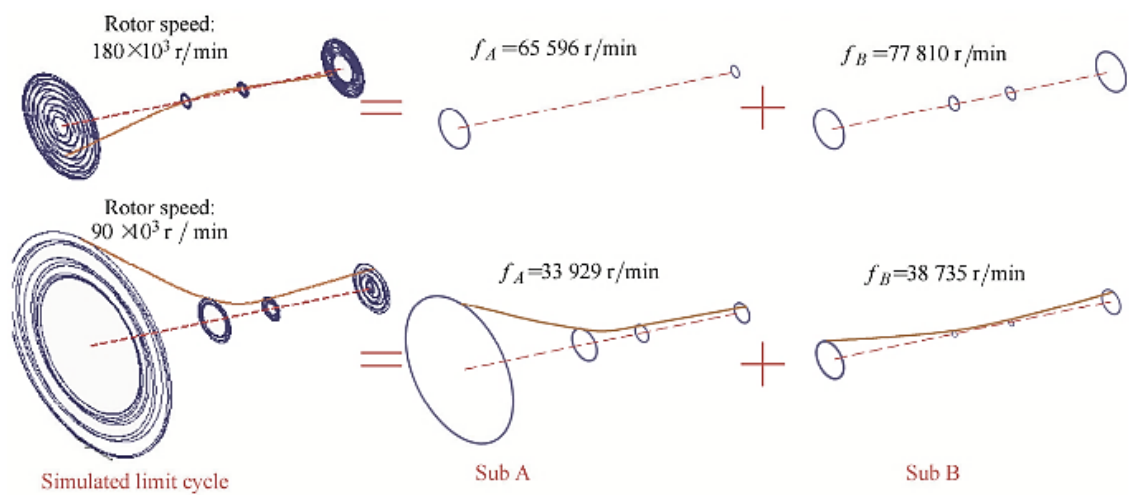

Figure 12. Rotor orbit at different rotational speed [21]

Figure 13 clearly shows that as the outer clearance increases, the changes in subsynchronous amplitudes are significant. When $c_{2}=24 \mu \mathrm{m}$, Sub 1 has a quite large amplitude while Sub 2 is hardly visible, and the limit cycles are perfect cycles. As $c_{2}$ increases, Sub 1 becomes weaker while Sub 2 extends downwards to 160,000 RPM. When $c_{2}$ continues to increase, Sub 1 weakens further and Sub 2 vibration occurs at lower and lower rotational speeds. At $c_{2}=40 \mu \mathrm{m}$, the magnitude of the subsynchronous vibrations are very similar to each other, and Sub 2 appears in almost the whole rotational speed range. When $c_{2}=46 \mu \mathrm{m}$, Sub 1 and Sub 2 merges to a new subsynchronous oscillation named Sub 0 . The combination starts from high rotor speeds and extends to lower rotor speeds like a „zipper”. When $c_{2}$ becomes $60 \mu \mathrm{m}$, the „zipper” pulls down to the low end of the rotor speed range, and Sub 0 starts at very low rotor speed, causing perfect cycles again [21, 27]. 


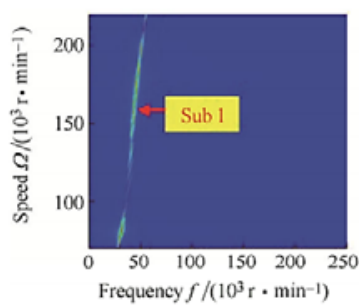

(a) $c_{2}=24 \mu \mathrm{m}$

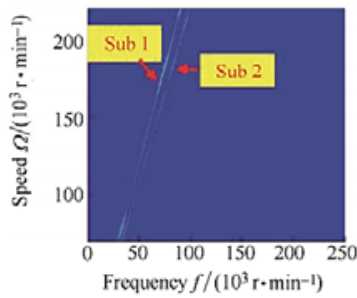

(d) $c_{2}=40 \mu \mathrm{m}$

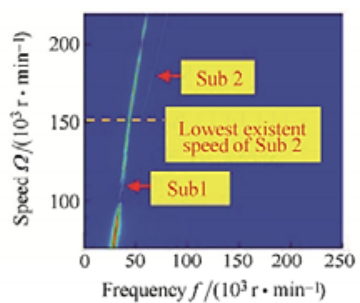

(b) $c_{2}=26 \mu \mathrm{m}$

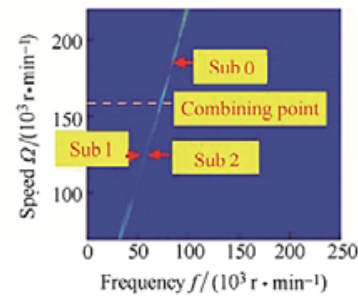

(e) $c_{2}=46 \mu \mathrm{m}$

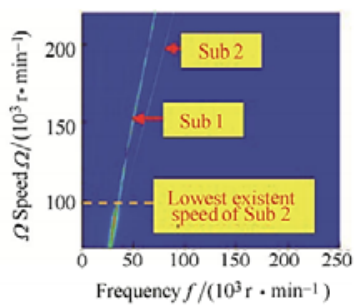

(c) $c_{2}=30 \mu \mathrm{m}$

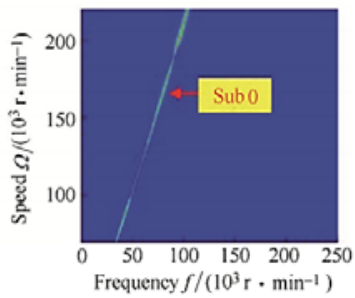

(f) $c_{2}=60 \mu \mathrm{m}$

Figure 13. Frequency contours in ideal condition [21]

Figure 14 shows the frequencies of Sub 0, 1 and 2 at 160,000 RPM with different outer clearances $\left(c_{2}\right)$. As the outer clearance increases, the subsynhcronous oscillations slowly move to 0.5 times the rotor speed frequency. The frequency of the Sub 1 vibration changes the most with the bearing clearance. Between 25 to 46 $\mu m$ outer clearance values, the frequency of Sub 1 increases from 0.285 to 0.456 times the rotor rotational speed. After the merge, the frequency of Sub 0 only slightly increases with the change in outer clearance $[21,26]$.

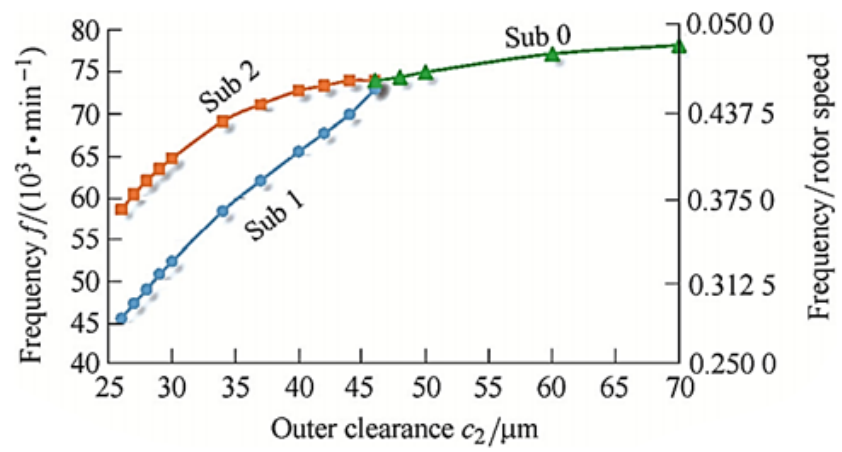

Figure 14. Frequencies of Sub 0, 1 and 2 at $160000 \mathrm{r} / \mathrm{min}$ with different $c_{2}$ [21] 


\subsection{Non-linear analysis of real conditions}

Under real conditions, the gravity and the unbalance always exist and have substantial influence to the behaviour of the rotor system. During this stage of the analysis, the gravity and unbalance effects were applied to the simulations [21].

The outer clearance $c_{2}$ was varied from 26 to $50 \mu \mathrm{m}$, and the rotor speed ranged from $70,000 R P M$ to $220,000 R P M$ in the simulation. Also, $0.04 \mathrm{~g} \cdot \mathrm{mm}$ unbalance was applied to the rotor (at both the compressor and the turbine side). In general, the subsynchronous oscillations go through a very similar development as the outer clearance increases like in the previous ideal simulation case. However, there are also some important differences between the ideal and real conditions. The evolution of Sub 2 is delayed. When $c_{2}=26 \mu \mathrm{m}$, Sub 2 only exists above 200,000 RPM under real conditions, while it was excited at about 160,000 RPM under ideal conditions. Also, Sub 2 changes with a greater extent compared to the ideal case, and the merging process happens at smaller outer clearance values. On the other hand, Sub 2 does not appear below 140,000 RPM under either conditions [21, 28].
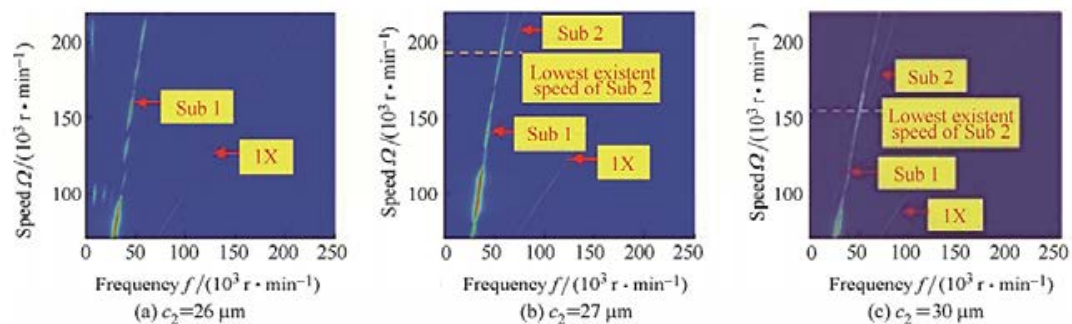

(b) $c_{2}=27 \mu \mathrm{m}$
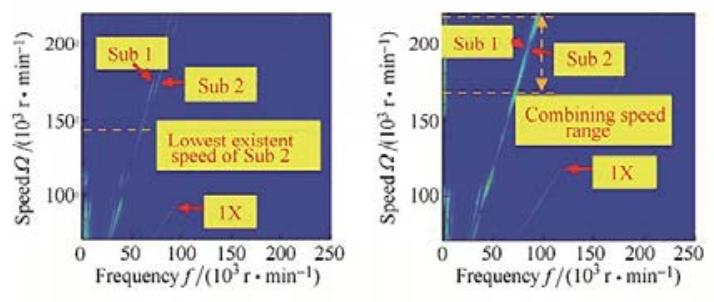

(c) $c_{2}=30 \mu \mathrm{m}$

(d) $c_{2}=40 \mu \mathrm{m}$

(e) $c_{2}=42 \mu \mathrm{m}$

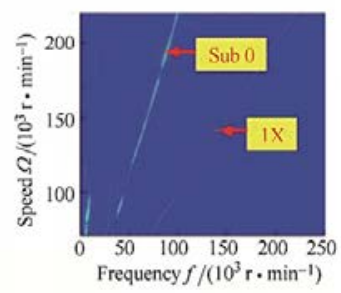

(f) $c_{2}=50 \mu \mathrm{m}$

Figure 15. frequency contours in real condition [21]

The explanation for these phenomena is that the rotor has two bearings and Sub 1 and Sub 2 occurs between two different bearing surfaces. Sub 1 occurs in bearing A and Sub 2 is in bearing B. In this case, the gravity centre of the rotor is very close to bearing $\mathrm{B}$, so the unbalance and the applied gravity has a greater influence on the Sub 2 oscillation [21]. 


\section{Implementation of pre-measurements on a compressed-air operating turbocharger component test-bench}

The comprehensive literature research provides an eligible amount of data to plan the turbocharger investigations, which leads to experimental tests of the simulation results. To successfully transplant the acquired information on the turbocharger rotrodynamics to measurements, the test-bench and the measuring system must be precise enough to detect the minor changes in the tribological system without disturbance. In such a way, pre-measurements are necessary before the experimental investigation of the bearing system, to ensure that the subsynchronous vibrations are measurable and the occurring noises on the test-bench are below the interference limit. In this purpose, vibration measurements were implemented on a compressedair test-bench to map the occurring noises and identify the turbocharger vibration components on a Campbell diagram.

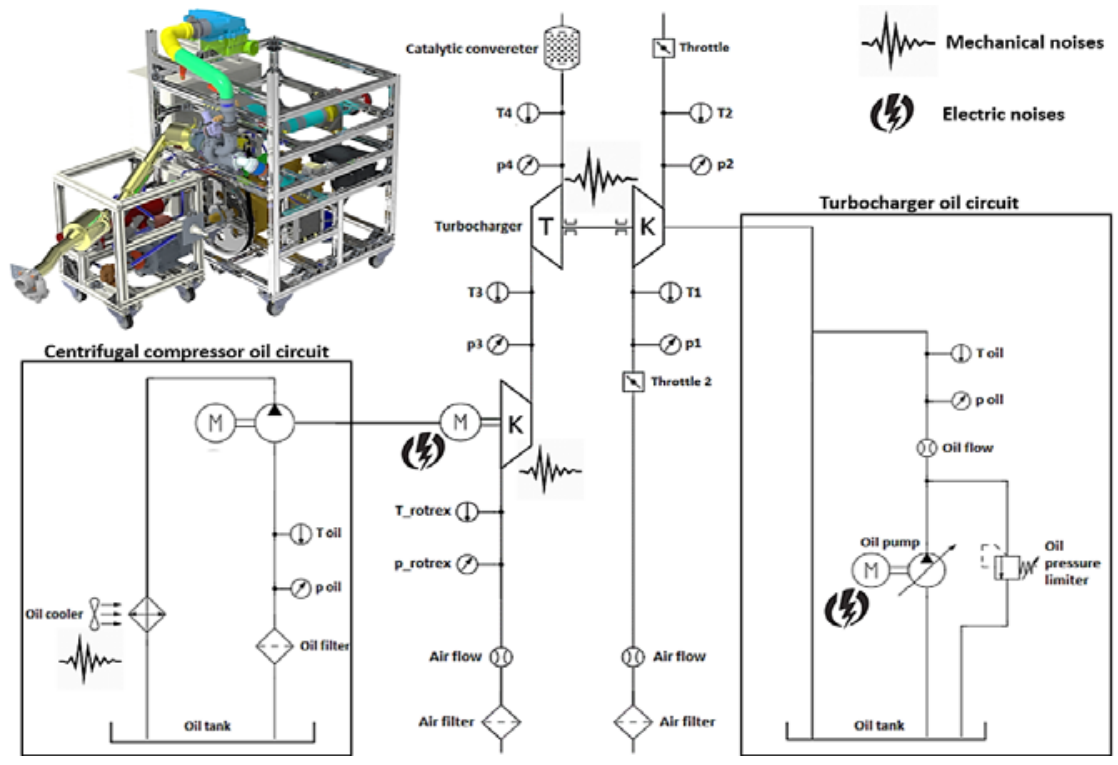

Figure 16. Structure and noises of the compressed-air test-bench

For the vibration analysis, an acceleration sensor has been mounted on the centerhousing of the turbocharger. The used sensor can measure even $\mu \mathrm{m} / \mathrm{s}^{2}$ accelerations, therefore, significantly low body-noises can cause unwanted signals. To ensure that the test results are appropriate and the interference factors are wellknown, the occurring noises of the test-bench (Figure 16.) itself had to be measured. An electric motor drives a centrifugal compressor with a high drive ratio. The 
rotating compressor produces the desired amount of air pressure to spin up the turbocharger rotor. The test-bench has a built-in oil circuit for the turbocharger with fully controllable oil inlet pressure. In addition to the observation of the equipment, the noises in the test room have been recorded, while the sensor was mounted on the turbocharger because the important information in terms of the vibration analysis is to determine the undesirable noises for the evaluation. The most dominant noises during operation are originating from the centrifugal compressor and the equipment's high voltage electric systems. The electric noises are emitted by the operation of the oil pump in the turbocharger oil circuit and from the electric motor which drives the centrifugal compressor.

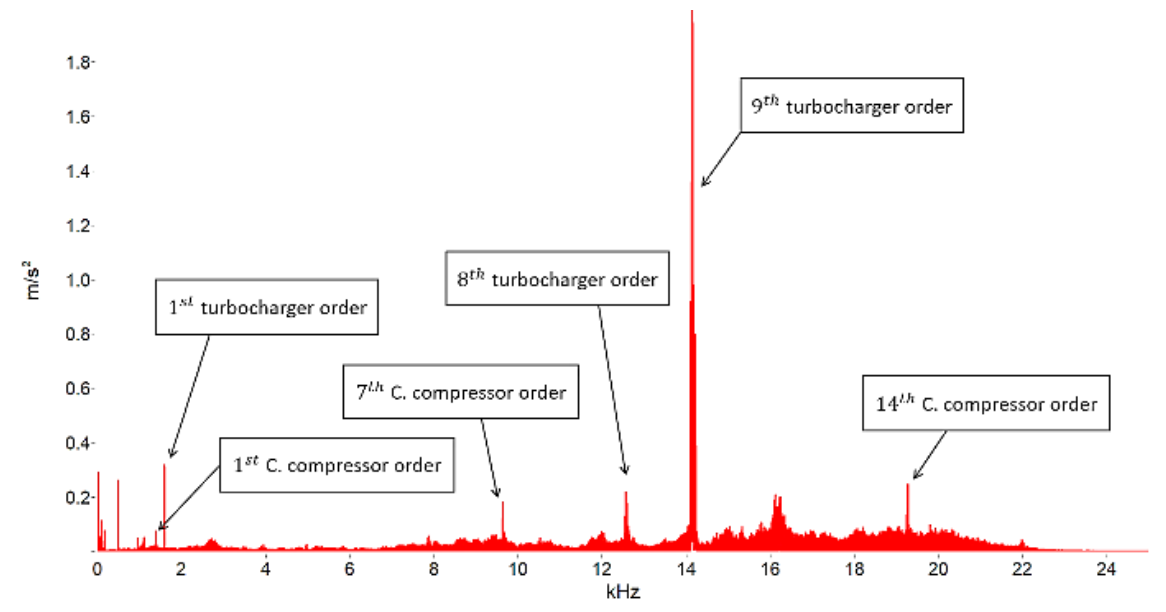

Figure 17. Fourier Spectrum of the measured turbocharger at stationary speed

Figure 17 shows the Fast Fourier Spectrum of the turbocharger. The rotational speed of the centrifugal compressor was set up to $84.000 R P M$, which corresponded to $100.000 R P M$ rotational speed on the turbocharger. The most dominant vibration components are marked on the diagram. As the figure shows, the highest amplitude vibration is around $14 \mathrm{kHz}$ frequency, which is the $9^{\text {th }}$ order of the turbocharger vibration (the vibration components of the turbocharger will be analyzed later in this chapter). The magnitude of the $9^{\text {th }}$ turbocharger order is close to $2 \mathrm{~m} / \mathrm{s}^{2}$, which is significantly higher than $7^{\text {th }}$ order of the centrifugal compressor's vibration. On the other hand, both the $7^{\text {th }}$ and the $14^{\text {th }}$ order of the centrifugal compressor are clearly visible on the spectrum of the turbocharger. This means, that the interference from the centrifugal compressor must be considered.

Figure 18 shows the Campbell diagram of the investigated turbocharger. The simplest vibration component to find on this spectrum is the first order (1), which 
equals the frequency of the rotor speed, e.g., if the rotational speed of the turbocharger is $60.000 R P M(10001 / s)$, then the first order or synchronous vibration occurs at $1000 \mathrm{~Hz}$. The cause of this vibration is mainly the unbalance of the rotor assembly of the turbocharger (compressor wheel, shaft, turbine wheel, compressor wheel nut). The implementation of a perfect balancing method is impossible, so the synchronous vibration component is always visible on the Campbell diagram under real circumstances

The oil whirling noise is marked with (2) on Figure 18, which lies below the frequency of the synchronous vibration This vibration is also called constant tone, due to its slight change in frequency on a run-up phase. In this spectrum, a jump can be seen in the frequency of the constant tone vibration at 104,000 RPM. The cause of this frequency jump is related to rotordynamic phenomena. It is presumed, that the vibration mode of the rotor system is changing from conical to cylindrical mode. The frequency of the oil whirl is highly dependent on the damping of the bearing system, which is affected by oil temperature, viscosity and the inner and outer radial bearing clearances.
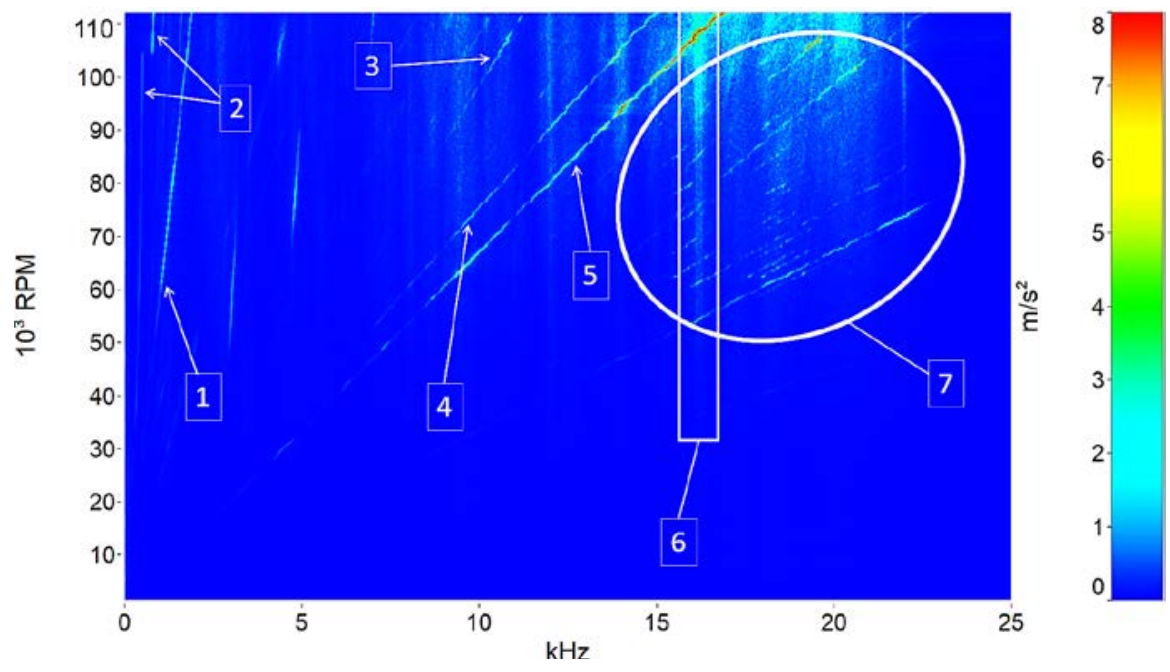

Figure 18. Turbocharger Campbell diagram

The vibration marked with 3 on Figure 18 shows the necessity of the measurements. The $7^{\text {th }}$ order vibration of the centrifugal compressor is clearly visible and could be disturbing in the evaluation of the measured data.

The component marked with 4 and 5 are the vane pass frequencies of the turbocharger compressor ( 8 vanes) and turbine (9 vanes) wheel. As mentioned in the 
previous chapter, the vane pass frequency can be obtained by multiplying the first order frequency with the number of vanes of the impeller. Vibration 4 and 5 are exactly 8 times and 9 times the synchronous vibration. The amplitudes of these vibrations are clearly affected by the rotational speed. Figure 18 shows that the $9^{\text {th }}$ order of the turbocharger has significantly higher amplitude at high rotor speeds (over $90.000 R P M$ ), than the $8^{\text {th }}$ order. This is believed to be a consequence of differing turbine and compressor wheel weight and geometry and the fact that the turbocharger is powered on the turbine side, but further measurements are needed to clarify the cause of this phenomenon. Impeller damage could increase the amplitude of the $1^{\text {st }}, 8^{\text {th }}$ and $9^{\text {th }}$, depending on the type of damage suffered.

Electric noises can be seen in the region marked with 6 on Figure 18. The electric noises can be easily recognized by the blurry appearance on the spectrum and the invariable frequency. As discussed in the previous chapter, the electric noises of the test-bench are located at $4 \mathrm{kHz}, 8 \mathrm{kHz}, 12 \mathrm{kHz}, 16 \mathrm{kHz}$ and $20 \mathrm{kHz}$. In this case, the most dominant components are the high-frequency electric noises, especially at $16 \mathrm{kHz}$. It is important to distinguish these noises from the vibrations of the turbocharger because these components influence the measured acceleration level and can be misleading without spectral analysis.

In the region marked with 7 , a group of high-frequency superharmonic components are visible. The frequency of these vibrations is the double of the vane pass components. The $14^{\text {th }}$ order of the centrifugal compressor vibration as well as the $16^{\text {th }}, 18^{\text {th }}$ turbocharger compressor and turbine vane pass orders are in this area of the Campbell diagram.

\section{Further potential in the topic of turbocharger vibration measurements as suggested by literature research}

Based on the studies presented and the test-bench measurements, there are several remaining questions in turbocharger design in terms of turbocharger vibro-acoustics, especially if viewed from experimental investigations side. It can be supposed that there always remains a difference between the simulations and the data measured on a test bench. The measurements should be developed from cold gas test benches to hot gas test benches, or even to engine test benches to ensure that test conditions are as close to the real operational environment as possible.

There are also plenty of major question in the topic of turbocharger rotordynamics, which should be investigated and measured to provide useful data for the further developments. The damping coefficient of the bearing system is depending on numerous factors, such as the geometrical parameters of the bearings and the shaft, the used material, the quality of the oil and temperature. To be able to measure the 
exact damping effect of the bearing system, a special tribometer is under development, which simulates the extremely high rotational speed of the rotor and measures the friction coefficient and the damping of the tribological system. For the investigations of the changing bending modes of the rotor under real conditions, high precision distance sensors are necessary. To be able to measure the motion of the shaft, the data processing and the sampling frequency of the measuring system needs to be very high. The shaft motion measuring system combined with vibration measurement can provide helpful information about the bending modes and the rotor response. With the mentioned equipment the magnitude of the constant tone vibration and the frequency jump of these oil whirl phenomena can be investigated.

\subsection{Measuring the effect of changing physical state of the oil on the vibrations caused by its whirl}

There is a huge potential in the vibration measurements with various oil quality and condition. Both temperature and viscosity level of the oil, as well as oil flow rate has an effect on the operation of the turbocharger rotor. Measuring the vibrations of the turbocharger with different oil temperatures can provide valuable information due to the change in damping coefficient of the oil, which depends on oil viscosity. With this measurement, vibrations at cold start and at hot operating conditions can be compared.

By the help of additional experimental investigations, results of simulations could further be validated and refined, thus reducing the costs of development phase of turbochargers. For better simulation results, the damping coefficient of oil could be calculated from test-bench measurements. By exciting the turbine shaft while accurately measuring acceleration levels, the damping coefficient can potentially be calculated, thus making the simulations more accurate. Under real operating conditions, damping coefficient of oil is also changing continuously as the oil is being heated up to operational temperature [29].

\subsection{Comparison of different bearing constructions}

Many of the publications studied dealt with the effect of bearing clearance dimensions change on characteristics of induced oscillations. A comprehensive experimental study could highlight the waterfall diagram differences between a semi-floating bearing and a full-floating bearing designs. With the use of cold gas and hot gas turbocharger test-benches in parallel, excited accelerations and characteristics of the subsynchronous vibrations could be validated in almost real operating conditions. 

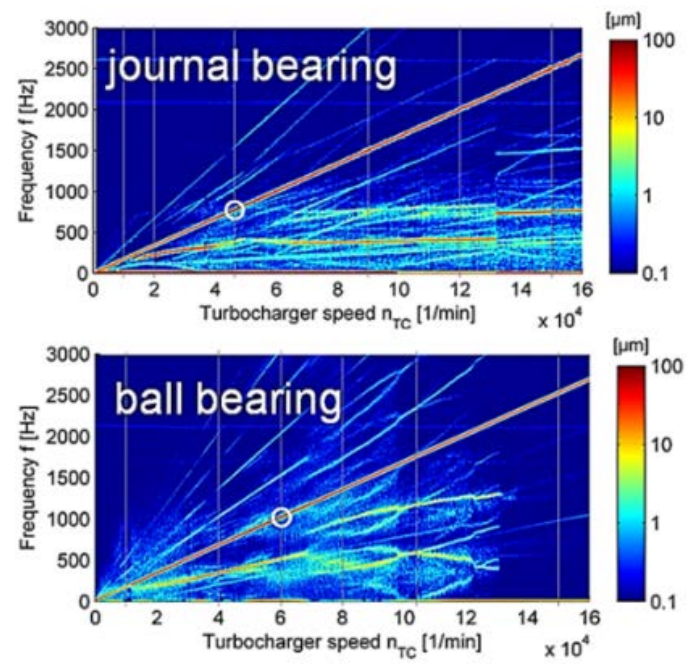

Figure 19. Campbell diagrams of turbochargers with different bearing constructions [30]

Ball bearings for automotive exhaust gas turbochargers are getting to become more common nowadays. Preliminary studies predict that better throttle response may be achieved by ball bearings due to the smaller friction forces at lower turbocharger rotational speeds. However, at high operating speeds, floating bearings also have less friction. Moreover, ball bearings have more intense acoustic emission levels, which negatively affects their penetration rate in automotive applications. Comparing the full-floating bearing, the semi-floating bearing and the ball bearing supported turbochargers on test-benches while measuring the emitted sound levels with microphones as well as the vibrations on the turbocharger in different operational conditions is important for the future of turbochargers. There are already some manufacturers that have tried ball bearing turbocharger applications in their vehicles [31].

\subsection{Research of vibrations caused by the deterioration of ideal bearing conditions}

Quality of the loaded bearing surfaces has strong effect on vibrations of the bearings. By measuring the acceleration levels on turbochargers with different bearing wear conditions a relation may be found between tribological phenomena inside the bearing housing and the vibrations generated outside. This method could be helpful for lifetime tests, or even could be a basis of an online damage detection system, supposing that the measurements are accurate and precise enough. To 
diagnose the surface condition, special confocal microscopes are currently used for recording the whole surface and even to evaluate roughness parameters or wear volume after every test run.
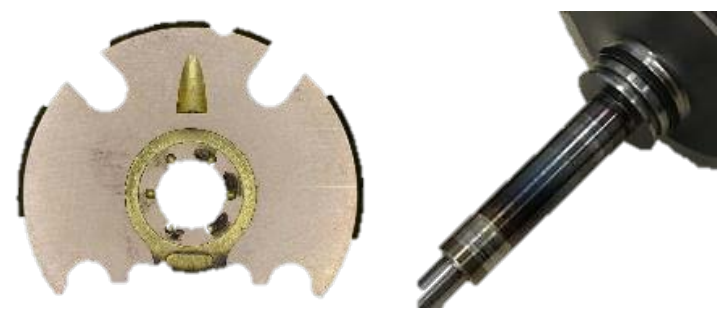

Figure 20. Wear on the loaded bearing surfaces of a turbocharger's thrust bearing and shaft

The aim of these measurements could primarily be the damage detection of the bearing system. By comparing turbocharger vibrations while the turbocharger is in assembled state with bearing conditions of different wear levels measured in disassembled state afterwards, functional dependences may be found to estimate bearing conditions online or real time. Early damage detection of the bearings thus may become possible, which would reduce time and cost of unnecessary disassembling of the chargers.

\section{Acknowledgement}

The paper was written with the support of the project titled "Internationalisation, initiatives to establish a new source of researchers and graduates and development of knowledge and technological transfer as instruments of intelligent specialisations at Széchenyi István University" (project number: EFOP-3.6.1-16-2016-00017).

\section{References}

[1] H. Nguyen-Schäfer, Rotordynamics of Automotive Turbochargers, $2^{\text {nd }}$ Edition, Springer International Publishing AG, Ludwigsburg, Germany, 2015. doi: https://dx.doi.org/10.1007/978-3-319-17644-4

[2] H. R. Born, Analytical and Experimental Investigation of the Stability of the Rotor-Bearing System of a New Small Turbocharger, ASME Paper No. 87GT-110, 1987. doi: https://doi.org/10.1115/87-GT-110 
[3] M. Tanaka, A Theoretical Analysis of Stability Characteristics of High Speed Floating Bush Bearings, Proceedings of 6th International Conference on Vibrations in Rotating Machinery, IMechE Conference Transaction 1996-6, Oxford, UK, Paper No. C500/087/96, pp. 133-142, 1996.

[4] L. San Andres, J. C. Rivadeneira, K. Gjika, C. Groves, G. LaRue, Rotordynamics of Small Turbochargers Supported on Floating Ring Bearings - Highlights in Bearing Analysis and Experimental Validation, Journal of Tribology 129 (2) (2007) pp. 391-397. doi: https://doi.org/10.1115/1.2464134

[5] C. Holt, L. San Andrés, Test Response of a Turbocharger Supported on Floating Ring Bearings Part I: Assessment of Subsynchronous Motions, Proceedings of 19th Biennial Conference on Mechanical Vibration and Noise, Chicago, IL, ASME Paper No. DETC 2003/VIB-48418, pp. 969-974, 2003. doi: https://doi.org/10.1115/DETC2003/VIB-48418

[6] L. San Andrés, J. Kerth, Thermal Effects on the Performance of Floating Ring Bearings for Turbochargers, Proceedings of the Institution of Mechanical Engineers, Part J: Journal of Engineering Tribology 218 (5) (2004) pp. 1-14. doi: https://doi.org/10.1243/1350650042128067

[7] M. Tanaka, Y. Hori, Stability Characteristics of Floating Bush Bearings, Journal of Lubrication Technolology 94 (3) (1972) pp. 248-259. doi: https://doi.org/10.1115/1.3451700

[8] J. Kerth, Prediction and Measurement of the Rotordynamic Response of an Automotive Turbocharger with Floating Ring Bearings, MSc thesis, Texas A\&M University, College Station, TX (2003).

URL http://hdl.handle.net/1969.1/ETD-TAMU-2003-THESIS-K426

[9] P. Bonello, Transient modal analysis of the non-linear dynamics of a turbocharger on floating ring bearings, Proceedings of the Institution of Mechanical Engineers, Part J: Journal of Engineering Tribology 223(1) (2009) pp. 79-93. doi: https://doi.org/10.1243/13506501JET436

[10] L. Tian, W. J. Wang, Z. J. Peng, Effects of bearing outer clearance on the dynamic behaviours of the full floating ring bearing supported turbocharger 
rotor, Mechanical Systems and Signal Processing 31 (2012) pp. 155-175. doi: https://doi.org/10.1016/j.ymssp.2012.03.017

[11] C. Li, Dynamics of rotor bearing systems supported by floating ring bearings, Journal of Lubrication Technology 104 (4) (1982) pp. 469-476. doi: https://doi.org/10.1115/1.3253258

[12] R. Holmes, M.J. Brennan, I. Gottrand, Vibration of an automotive turbocharger-a case study, $8^{\text {th }}$ International Conference on Vibrations in Rotating Machinery, Institution of Mechanical Engineers, Swansea, UK, pp. 445-455, 2004.

[13] M. Hemmi, T. Inoue, Effects of characteristics of floating bush bearings on the stability of turbochargers, The Proceedings of the International Conference on Motion and Vibration Control, Saitama, Japan, pp. 979-984, 2002.

doi: https://doi.org/10.1299/jsmeintmovic.6.2.979

[14] R. Kirk, A.A. Alsaeed, Induced unbalance as a method for improving the dynamic stability of high speed turbochargers, International Journal of Rotating Machinery, Article ID 952869, p. 9, 2011. doi: http://dx.doi.org/10.1155/2011/952869

[15] P. Kamesh, Oil-Whirl instability in an Automotive Turbocharger, $\mathrm{PhD}$ Thesis, University of Southampton, Faculty of Engineering and the Environment, Institute of Sound and Vibration Research (2011). URL https://eprints.soton.ac.uk/188121/1/188121P2767.pdf

[16] W. Köhl, M. Kreschel, D. Filsinger, Experimental and numerical investigations on an automotive turbocharger with a transparent bearing section, $11^{\text {th }}$ International Conference on Turbochargers and Turbocharging, pp. 349-359, 2014.

doi: https://doi.org/10.1533/978081000342.349

[17] B. Schweizer, M. Sievert, Nonlinear oscillations of automotive turbocharger turbines. Journal of Sound and Vibration 321 (3-5) (2009) pp. 955-975. doi: https://doi.org/10.1016/j.jsv.2008.10.013

[18] L. San Andres, J. Rivadeneira, K. Gjika, A virtual tool for prediction of turbocharger nonlinear dynamic response: validation against test data, Journal 
of Engineering for Gas Turbines and Power 129 (4) (2006) pp. 1035-1046. doi: https://doi.org/10.1115/1.2436573

[19] B. Schweizer, Total instability of turbochargers rotors - Physical explanation of the dynamic failure of rotors with full-floating ring bearings, Journal of Sound and Vibration 328 (1-2) (2009) pp. 156-190. doi: https://doi.org/10.1016/j.jsv.2009.03.028

[20] B. Schweizer, Oil whirl, oil whip and whirl/whip synchronization occurring in rotor systems with full-floating ring bearings, Nonlinear Dynamics 57 (4) (2009) pp. 509-532.

doi: https://doi.org/10.1007/s11071-009-9466-3

[21] L. Feng, Z. Ming, X. Quanyong, Effects of Semi-floating Ring Bearing Outer Clearance on the Subsynchronous Oscillation of Turbocharger Rotor, Chinese Journal of Mechanical Engineering 29 (5) (2016) pp. 901-910. doi: https://doi.org/10.3901/CJME.2016.0421.057

[22] L. Chaofeng, Y. Hexing, Z. Shihua, Simulations and experimental investigation on motion stability of a flexible rotor-bearing system with a transverse crack, Chinese Journal of Mechanical Engineering 26 (6) (2013) pp. 1194-1203.

doi: https://doi.org/10.3901/CJME.2013.06.1194

[23] P. Bonello, M. J. Brennan, R. Holmes, Non-linear modelling of rotor dynamic systems with squeeze film dampers - an efficient integrated approach, Journal of Sound and Vibration 249 (4) (2002) pp. 743-773.

doi: https://doi.org/10.1006/jsvi.2001.3911

[24] S. T. Noah, P. Sundararajan, Significance of considering nonlinear effects in predicting the dynamic behavior of rotating machinery, Journal of Vibration and Control 1 (4) (1995) pp. 431-458.

doi: https://doi.org/10.1177/107754639500100403

[25] R. I. Taylor, The inclusion of lubricant shear thinning in the short bearing approximation, Proceedings of the Institution of Mechanical Engineers, Part J: Journal of Engineering Tribology 213 (1) (1999) pp. 35-46. doi: https://doi.org/10.1243/1350650991542596

[26] K. Gjika, L. San Andrés, G. D. Larue, Nonlinear dynamic behavior of turbocharger rotor-bearing systems with hydrodynamic oil film and squeeze 
film damper in series: prediction and experiment, Journal of Computational and Nonlinear Dynamics, 2010, 5(4): 041006.

doi: https://doi.org/10.1115/1.4001817

[27] C. Weng Jen, Rotordynamics and bearing design of turbochargers, Mechanical Systems and Signal Processing, 29 (2012) pp. 77-89. doi: https://doi.org/10.1016/j.ymssp.2011.07.025

[28] W. Zheng, W. Zengquan, Z. Li, Time-dependent vibration frequency reliability analysis of blade vibration of compressor wheel of turbocharger for vehicle application, Chinese Journal of Mechanical Engineering, 27 (1) (2014) pp. 205-210. doi: https://doi.org/10.3901/CJME.2014.01.205

[29] J.W. Lund, E. Saibel, Oil Whip Whirl Orbits of a Rotor in Sleeve Bearings, Journal of Engineering for Industry, 89 (4) (1967) pp. 813-823. doi: https://doi.org/10.1115/1.3610159

[30] C. Biet, R. Baar, Experimental study on ball bearings for exhaust gas turbocharger, 5th Tribology and Effeciency Conference of Györ, pp. 253272, 2018.

[31] D. Zeppei, S. Koch, A. Rohi, Wälzlagerungstechnologie für Pkw-Turbolader, MTZ - Motortechnische Zeitschrift 77 (11) (2016) pp. 28-35. doi: https://doi.org/10.1007/s35146-016-0112-6 